\title{
A Comprehensive Study of Guanine Excited State Relaxation and Photoreactivity in G-Quadruplexes
}

Lara Martinez-Fernandez ${ }^{1,2^{*}}$ Pascale Changenet, ${ }^{1,3}$ Akos Banyasz, ${ }^{1}+$ Thomas Gustavsson, ${ }^{1}$

Dimitra Markovitsi ${ }^{1 *}$ and Roberto Improta ${ }^{1.4}$

\section{AUTHOR ADDRESS}

Lara Martinez-Fernandez ${ }^{1 \xi^{*}}$ Pascale Changenet, ${ }^{1,2}$ Akos Banyasz, ${ }^{1 \dagger}$ Thomas Gustavsson, ${ }^{1}$

Dimitra Markovitsi ${ }^{1^{*}}$ and Roberto Improta ${ }^{1,3}$

\section{AUTHOR ADDRESS}

1. LIDYL, CEA, CNRS, Université Paris-Saclay, F-91191 Gif-sur-Yvette, France

2. Laboratoire d'Optique et Biosciences, Institut Polytechnique de Paris, CNRS, INSERM, Université Paris-Saclay, 91128 Palaiseau cedex, France

3. Istituto Biostrutture e Bioimmagini- Consiglio Nazionale delle Ricerche, Via Mezzocannone 16, I-80134 Napoli, Italy 
$\begin{array}{lr}\text { A. Experimental Details } & 2\end{array}$

B. Computational Details and Results 3-5

$\begin{array}{lr}\text { C. Figures and Tables } & \text { 6-15 }\end{array}$ 


\section{A. Experimental Details}

TEL21 oligomers were purchased from Eurogentec Europe; they were purified by HPLC and heir purity was tested by MALDI-TOF. The lyophilized powder was dissolved in phosphate buffer $\left(0.03 \mathrm{molL}^{-1}\right.$

$\mathrm{Na}_{2} \mathrm{HPO}_{4}, 0.03 \mathrm{molL}^{-1} \mathrm{Na}_{2} \mathrm{PO}_{4}, 0.48 \mathrm{molL}^{-1} \mathrm{NaCl}$ ). The purity of the buffer ingredients (Fluka) was higher than $99.999 \%$. TEL21/ $\mathrm{Na}^{+}$structures were prepared using a dry bath (Eppendorf-ThermoStatplus); $2 \mathrm{~mL}$ of concentrated solution were heated up to $96^{\circ} \mathrm{C}$ during $5 \mathrm{~min}$, cooled down slowly to $4^{\circ} \mathrm{C}$ and maintained at this temperature overnight. Subsequently, mother solutions were diluted according to requirements of various experiments which were performed at $20-23^{\circ} \mathrm{C}$.

Steady-state absorption and fluorescence spectra were obtained, respectively, with a Perkin Lambda 900 spectrophotometer and a Jobin-Yvon Fluorolog-3 spectrofluorimeter. Fluorescence spectra were recorded at right-angle configuration and corrected for the response of the detection system; the absorbance at the excitation wavelength was lower than 0.1 . The fluorescence quantum yields were determined using TMP as a reference. ${ }^{1}$

The excitation source of the time-resolved experiments was the third harmonic $(267 \mathrm{~nm}, 120 \mathrm{fs})$ of a TiSapphire laser (Coherent MIRA 900). The FU setup is described in detail elsewhere. ${ }^{2}$ The TCSPC setup used a Becker \& Hickl GmbH PC card and a microchannel plate (R1564 U Hamamatsu). The instrumental

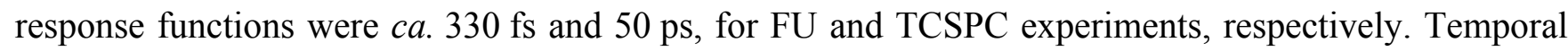
scans were made for parallel $\left(I_{p a r}\right)$ and perpendicular $\left(I_{\text {perp }}\right)$ excitation-detection configurations by controlling the polarization of the exciting beam with a half-wave plate. The total fluorescence intensity $F(t)$ and the fluorescence anisotropies $r(t)$ were constructed according to the equations:

$F(t)=I_{\text {par }}(t)+2(t)$ and $r(t)=\left(I_{\text {par }}(t)-I_{\text {perp }}(t)\right) /\left(I_{\text {par }}(t)+2 I_{\text {perp }}(t)\right)$.

Special cautions were taken to eliminate the contribution of photoproducts to the fluorescence decays. For FU (peak intensity: $0.2 \mathrm{GW} \mathrm{cm}^{-2}$ ), $25 \mathrm{~mL}$ of solution was circulating through a flow-cell (optical pathlength: $1 \mathrm{~mm}$ ) whereas for TCSPC (peak intensity: $3 \mathrm{kWcm}^{-2}$ ), ca. $3 \mathrm{~mL}$ of solution contained in $10 \mathrm{~mm}$ $\times 10 \mathrm{~mm}$ quartz cells was continuously stirred. Successive measurements gave identical decays which were eventually merged to increase the signal-to-noise ratio.

Irradiations at $266 \mathrm{~nm}$ were performed using the fourth harmonic of a Nd:YAG laser (Spectra-Physics, Quanta Ray) with an excitation frequency of $0.2 \mathrm{~Hz}$. The solution $(2 \mathrm{~mL}$, absorbance at $266 \mathrm{~nm}$ equal to 2) was contained in $1 \mathrm{~cm} \times 1 \mathrm{~cm}$ quartz cells and mildly stirred; its temperature was maintained at $20^{\circ} \mathrm{C}$. The energy of the exciting pulse, was measured by a NIST traced pyroelectric sensor (OPHIR Nova2/PE25). 


\section{B. Computational Details and Results}

\section{$\underline{\text { Models }}$}

Two different theoretical models (Figure SI-1) were used to described Tel21/ $\mathrm{Na}^{+}$starting from the NMR structure reported for the human telomeric sequence (d[AG3(T2AG3)3]). ${ }^{3}$. In Model1 we consider the whole G4 structure, that is, the guanine core, the loops, the inner and outer counter ions $\left(\mathrm{Na}^{+}\right)$. In Model2 we focus on the guanine core and the ions, without the loops. Absorption and excited state optimizations were carried out for both models leading to equivalent results (see below). However, due to the large size of Model1, containing more than 700 atoms, part of our analysis, such as the determination of electronic densities and the dimerization process, was performed with Model2. When we refer to the mixture of the noninteracting monomers, we consider 12 dGMP + 1TMP as found in Model 1 and 12 dGMP for Model2.

\section{Density Functional Theory (DFT) calculations}

Ground and excited states were optimized using Quantum Mechanics/Molecular Mechanics (QM/MM) calculations. Density Functional Theory and its time dependent version was used for the calculation of the QM part, selecting the M052X4 functional and 6-31G(d) basis set. We selected the M052X because it allows an accurate treatment of dispersion interactions and, therefore, it is suitable to study stacked systems. Moreover, it provides a reliable description of CT transitions, ${ }^{5-7}$ in good agreement with the results obtained on Wave-Function based methods. As a consequence, it has been already profitably used in the study of strongly coupled multi-chromophore system as DNA, and in particular G4, as shown by many previous studies 
concerning photoionization, photophysics or photochemistry. ${ }^{8-10}$ Contrary, the MM part was described using the Amber parm96.dat ${ }^{11}$ force fields. Both parts were coupled using the ONIOM $^{12}$ procedure as implemented in Gaussian09.13 The whole QM/MM system was considered in solvation (water) using Linear response Polarizable Continuum Model (PCM), ${ }^{14}$, 15 and taking into account solvent dynamics through State Specific ${ }^{16,17}$ calculations when specified.

\section{Functional and basis set}

We checked the dependence of our results with the selected functional and basis set by

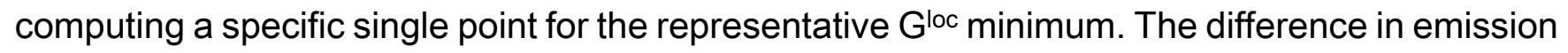
energies are less than $2 \%$, being $4.44 \mathrm{eV}$ at M052X/6-31G(d), $4.40 \mathrm{eV}$ at CAM-B3LYP/6-31G(d) and $4.34 \mathrm{eV}$ M052X/ 6-311+G(d,p) levels of theory. The oscillator strength at CAM-B3LYP/6$31 G(d)$ is very similar and the stability of $(G-G)^{\text {deloc }}$ compared to $G^{\text {loc }}$ is of the same order of magnitude but slightly larger ( $\sim 0.8 \mathrm{eV}$ vs $\sim 0.6$ at M052X QM level). It must be considered that, however, the enlarging of the basis set involves a $\sim 20$ fold increase of the computational cost.

\section{Energies and absorption spectra}

For better comparison with the experimental spectra, each transition was convoluted by a Gaussian curve (0.3 eV hwhm). Vertical excitation energies are not expected to necessarily correspond to the absorption maxima of the experimental spectra depending also on vibrational and thermal effects. It was shown in previous studies that, since the excited state frequencies 
are lower than the ground state ones, we can expect a systematic red-shift $(0.1-0.3 \mathrm{eV})$ of the computed absorption maximum with respect to the vertical excitation energy. ${ }^{18}$ Since the calculation of a vibrational resolved spectrum is not feasible for TEL21/Na+, we simply redshifted all the computed vertical absorption energies by a single value, i.e. $0.6 \mathrm{eV}$, which accounts for various possible source of errors (method, functional, basis set etc), in addition to the lack of vibrational and thermal effects. Such a value allows the superposition of the computed vertical excitation energy with the experimental absorption maximum of dGMP in water at the same level of theory $(M 052 X / 6-31 G(d))$. This value have been used in previous studies to compare computed absorption spectra with experimental ones obtained for guanine and guanine radicals. ${ }^{8}$ However, we are aware that different sources of errors in our calculations would need different corrections for all various excited states here considered. On the other hand, this shift enables to appreciate what is the effect of the formation of the quadruplex on the absorption spectra of guanine (since all the spectra are shifted of the same value). Vibrational effects are instead expected to blue-shift the fluorescence spectra with respect to the computed vertical emission energies, i.e. in the opposite direction with respect the other sources of computational error. As a consequence, we have not applied any correction to emission energies.

\section{$\underline{(G-G)^{C T} \text { minima }}$}

Since LR-PCM underestimates the stability of charge transfer (CT) excited states in polar solution, it has not been possible to optimize the minima of $(G-G)^{C T}$ by using M052X functional, which correctly describes the stability of CT states in the gas phase. The solvent stabilization of 
CT states in water is instead more correctly described by State Specific (SS)-PCM calculations, which, however, cannot be used in geometry optimizations. In order to circumvent this problem we resorted to a procedure profitably adopted in the past, using PBE0, ${ }^{19}, 20$ which is biased to traditional deficiencies of standard DFT functional and, at difference of M052X, overestimates the stability of CT states. As a consequence, it is possible to locate CT minima also by using LR-PCM. We thus, optimizing $(G-G)^{C T}$ using the PBE0 functional. From there, trials to reoptimize it using M052X were unsuccessful, decaying to most stable (G-G) deloc minimum. Constrained optimizations, with the two guanines frozen to the PBE0 geometry, led to a minimum which is only $\sim 0.5 \mathrm{eV}$ less stable than $(\mathrm{G}-\mathrm{G})^{\text {deloc }}$.

\section{Franck Condon states}

All the states in the "low energy" region have a $\pi \pi^{*}$ character and are related to the La excited state of the dGMP, whose transition energy $(\Delta E)$ and oscillator strength (f), computed at the same level of theory, are $4.77 \mathrm{eV}$ and 0.19 , respectively. They mainly differ in their degree of delocalization among guanine bases (Figure $\mathrm{SI}-3)$. For the lowest ones $\left(\mathrm{S}_{1}\right.$ to $\mathrm{S}_{3} ; \Delta \mathrm{E}=4.3-4.4$ $\mathrm{eV}$ and $\mathrm{f}<0.1$ ), the excitation is mainly localized on a single guanine and only a small fraction of the electronic density is shared with a neighboring vertically stacked guanine. When delocalization occurs among non-vertically stacked guanines, i.e guanines in different tetrads and strands but with the closest strand, the $\mathrm{f}$ is less affected, as, for example, in the case of $\mathrm{S}_{4}$ $(\mathrm{f}>0.1, \Delta \mathrm{E}=4.43 \mathrm{eV}) . \mathrm{S}_{5}$ and $\mathrm{S}_{9}$, are characterized by higher degree of delocalization, up to 3

or 4 bases, and significantly lower $f\left(10^{-2}\right)$. In contrast, much larger oscillator strength is found 
for completely localized states, such as $S_{10}(f=0.20,4.56 \mathrm{eV})$. For all these excited states, the CT character, computed using the Mulliken charges $(\delta q)$, is smaller than 0.05 a.u. The spectra computed for Model1 and Model2 are almost identical (Figure SI-5).

The high energy region of the spectra, and thereby the absorption maximum, is also due to $\pi \pi^{*}$ excited states but resembling to Lb excited states of dGMP $(5.24 \mathrm{eV}, \mathrm{f}=0.43)$. In the case of TEL21/Na $\mathrm{Na}^{+}$there are several states with such nature being the first one $\mathrm{S}_{18}$, with energies in the 5.0-5.2 $\mathrm{eV}$ range and very large $\mathrm{f}=0.5-1$. Delocalization is also possible for these states (Figure SI-4)

To better understand the specific features of Tel21/Na+ absorption spectrum, we performed a similar theoretical analysis for (i) a GGG single strand, (ii) a G tetrad, and (iii) Model1 and Model2 without $\mathrm{Na}^{+}$ions in the central cavity keeping the same geometry as for the optimized G4 (Figure SI-5, 6 and 7). We found that the hyperchromism at the low energy part of the spectrum is significantly reduced in the absence of $\mathrm{Na}^{+}$ions.

\section{Charge transfer character}

We have estimated the charge transfer character computing the difference between the Mulliken or the natural charges of the ground state at $\mathrm{FC}(\mathrm{qFC})$ and of the excited state at the minima (qS1):

$\delta q=q S 1-q F C$

When Mulliken charges are used, we obtained that the CT character for $(\mathrm{G}-\mathrm{T})^{\mathrm{CT}}$ and $(\mathrm{G}-\mathrm{G})^{\mathrm{CT}}$ minima are 0.8 and 0.7 a.u, respectively. In case of Natural charges the resulting numbers are 
0.9 and 0.6 a.u. It can be then concluded that both minima have an important CT character, independently of the computational charge model. 


\section{Figures and Tables}
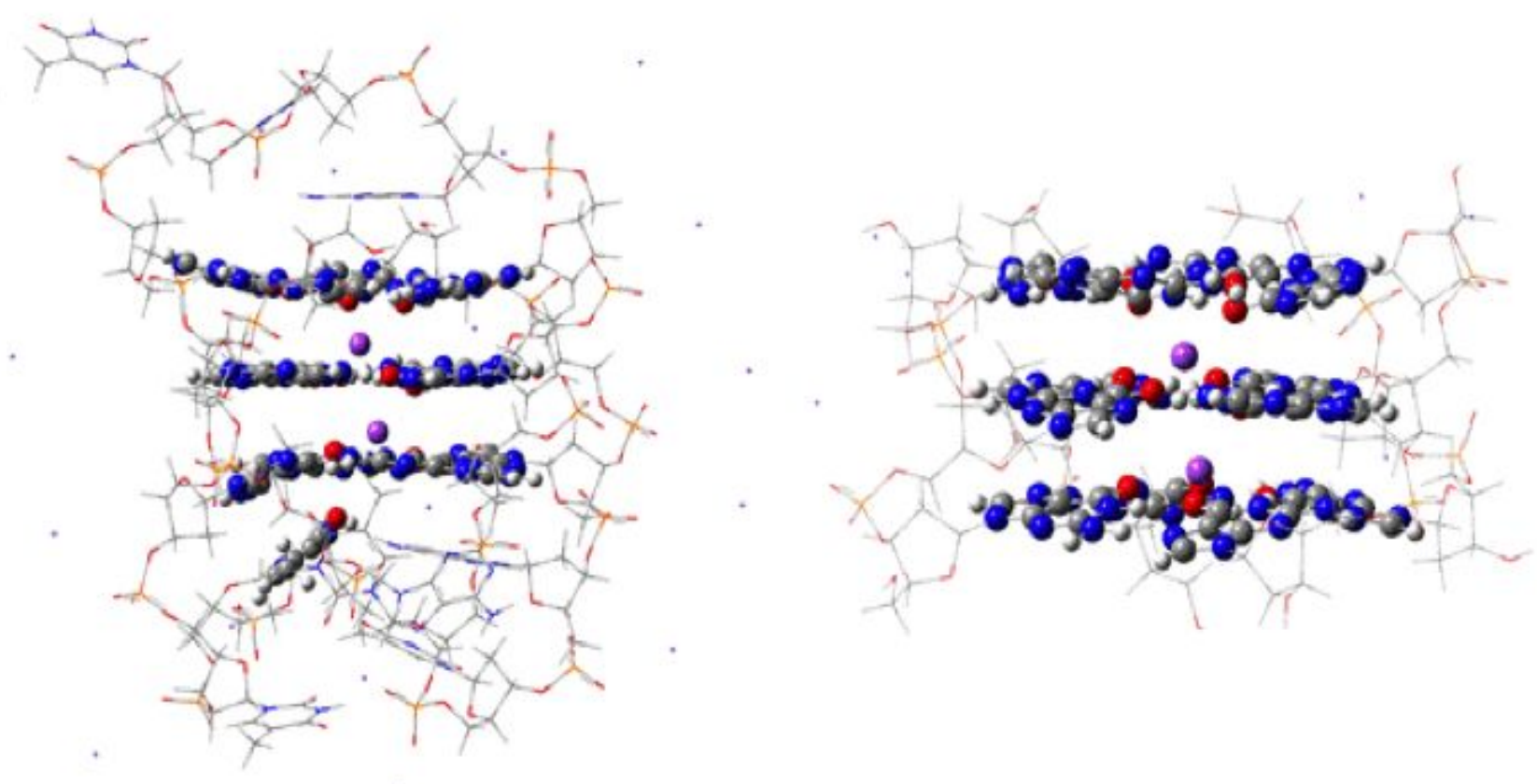

Figure SI-1 Model1 (left) and Model2 (right) used for the calculations. Atoms treated at the QM level are depicted in ball \& sticks and the groups described at the MM level in lines.

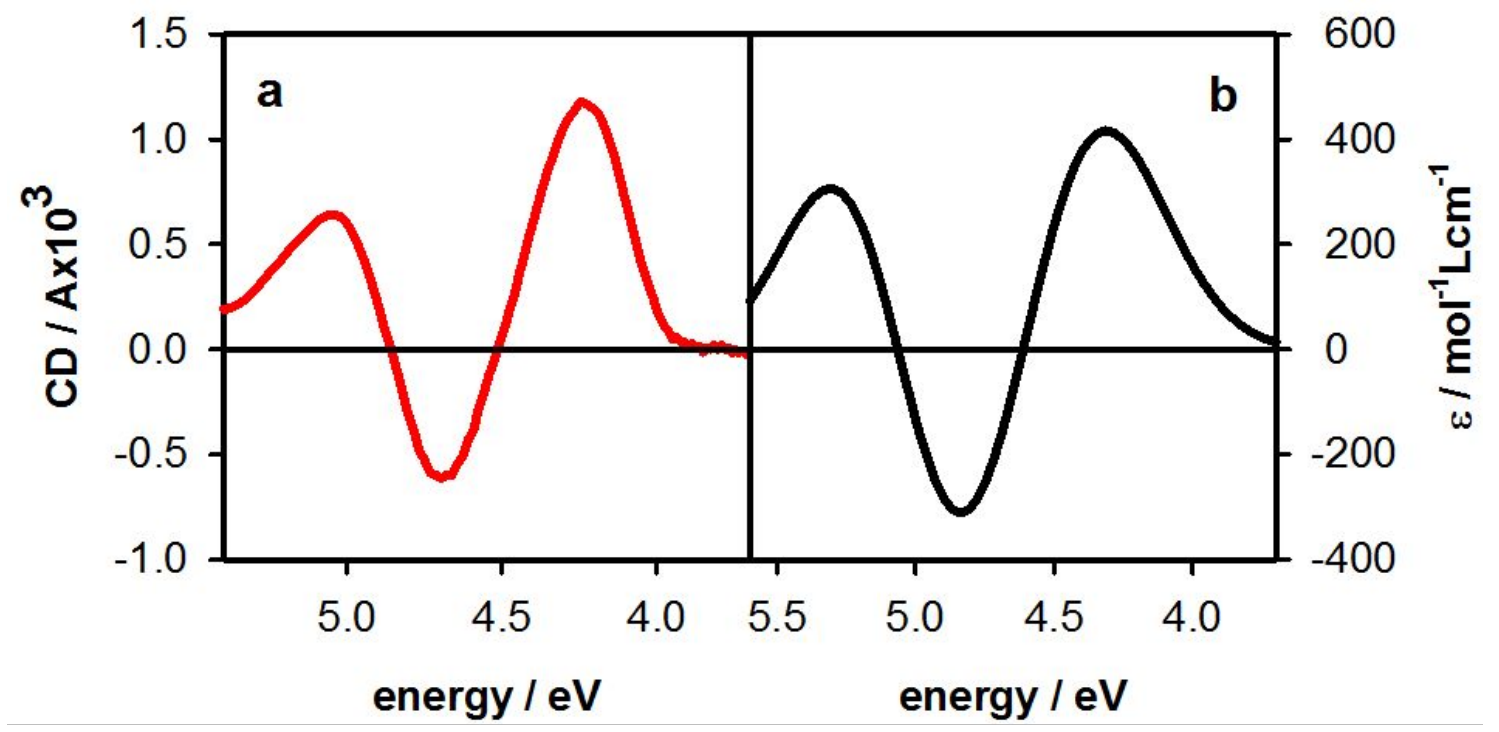


Figure SI-2. Experimental (a) and computed (b) circular dichroism spectra. The computed transitions were convoluted by Gaussian curves with $0.6 \mathrm{eV}$ (fwhm) and their energy was shifted by $-0.6 \mathrm{eV}$. 
Table SI-1. Main character (less important character shown within second row), shifted vertical absorption energies and oscillator strengths for the excited states of Tel21 (Model2) below 4.60 $\mathrm{eV}$. See Scheme 1 in the main text for $\mathrm{G}$ numbering.

\begin{tabular}{|c|c|c|c|c|c|c|c|}
\hline & Charc. & $\begin{array}{l}\Delta \mathrm{E} \\
\mathrm{eV}\end{array}$ & $f$ & & Charc. & $\begin{array}{l}\Delta \mathrm{E} \\
\mathrm{eV}\end{array}$ & $f$ \\
\hline \multirow[t]{2}{*}{$S_{1}$} & $\mathrm{G}^{\prime} \rightarrow \mathrm{G}^{\prime *}$ & 4.31 & 0.07 & $\mathrm{~S}_{6}$ & $\mathrm{G} 3 \rightarrow \mathrm{G}^{*}$ & 4.49 & 0.13 \\
\hline & G3" $\rightarrow$ G3"* & & & & $\mathrm{G} 1 \rightarrow \mathrm{G} 1^{*}$ & & \\
\hline \multirow[t]{2}{*}{$\mathrm{S}_{2}$} & $\mathrm{G} 4^{\prime} \rightarrow \mathrm{G}^{\prime *}$ & 4.35 & 0.08 & $S_{7}$ & $\mathrm{G} 1 \rightarrow \mathrm{G} 1^{*}$ & 4.50 & 0.11 \\
\hline & G4" $\rightarrow$ G4"* & & & & $\mathrm{G} 2 \rightarrow \mathrm{G} 2^{*}$ & & \\
\hline \multirow[t]{2}{*}{$\mathrm{S}_{3}$} & $\mathrm{G} 2^{\prime} \rightarrow \mathrm{G}^{\prime *}$ & 4.38 & 0.10 & $\mathrm{~S}_{8}$ & $G 1 " \rightarrow G 1$ "* & 4.52 & 0.12 \\
\hline & $\mathrm{G} 2 " \rightarrow \mathrm{G} 2$ "** & & & & & & \\
\hline \multirow[t]{4}{*}{$\mathrm{S}_{4}$} & $\mathrm{G} 1^{\prime} \rightarrow \mathrm{G} 1^{\prime *}$ & 4.43 & 0.16 & $\mathrm{~S}_{9}$ & G3" $\rightarrow G 3 " *$ & 4.53 & 0.05 \\
\hline & $\mathrm{G} 4 \rightarrow \mathrm{G} 4^{*}$ & & & & $G 4 " \rightarrow G 4 " *$ & & \\
\hline & & & & & $\mathrm{G} 4 \rightarrow \mathrm{G} 4^{*}$ & & \\
\hline & & & & & $\mathrm{G} 1 \rightarrow \mathrm{G} 1^{*}$ & & \\
\hline \multirow[t]{4}{*}{$\mathrm{S}_{5}$} & $\mathrm{G} 1^{\prime} \rightarrow \mathrm{G} 1^{\prime *}$ & 4.45 & 0.01 & $S_{10}$ & G4" $\rightarrow G 4 " *$ & 4.56 & 0.27 \\
\hline & $\mathrm{G} 4 \rightarrow \mathrm{G} 4^{*}$ & & & & & & \\
\hline & $\mathrm{G} 1 \rightarrow \mathrm{G} 1^{*}$ & & & & & & \\
\hline & & & & $S_{11}$ & $\mathrm{G} 1 " \rightarrow \mathrm{G} 1$ "* & 4.60 & 0.29 \\
\hline
\end{tabular}




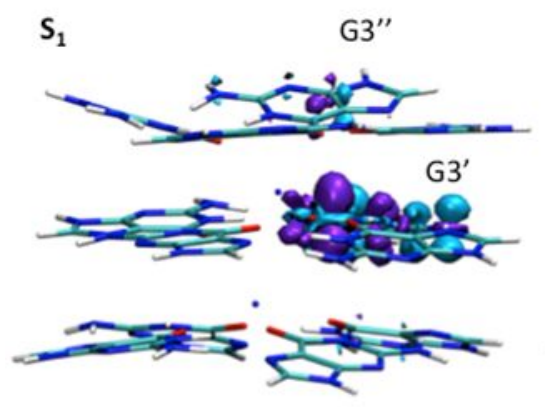

$S_{3}$
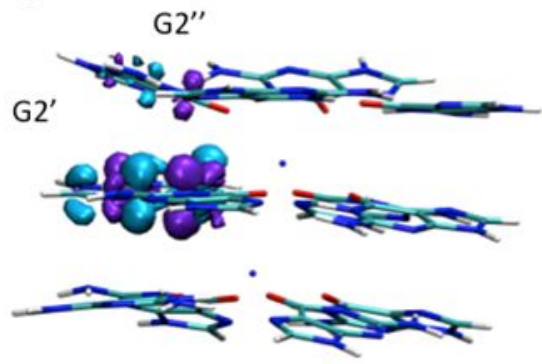

$\mathrm{S}_{5}$

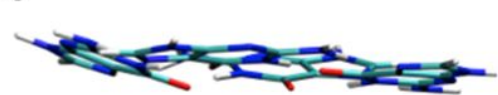

G1'

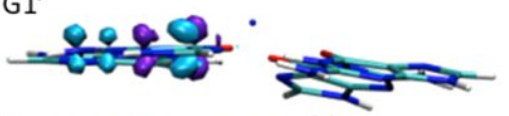

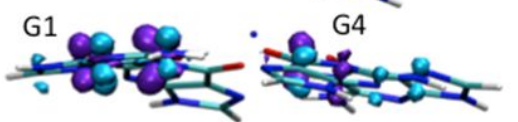

S
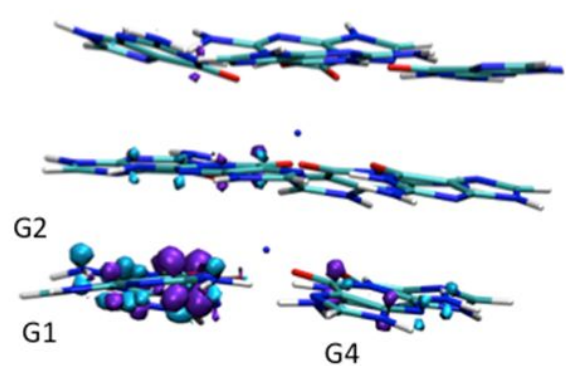

S
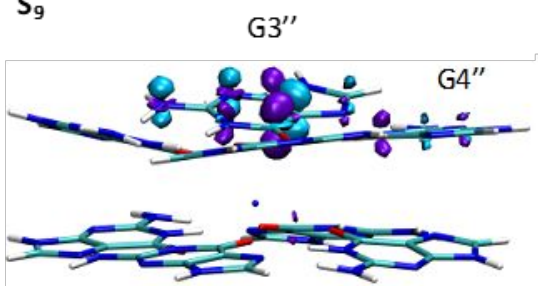

G4

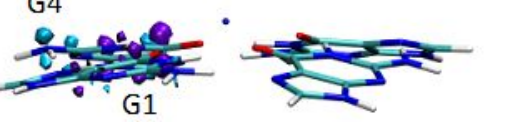

$\mathrm{S}_{2}$
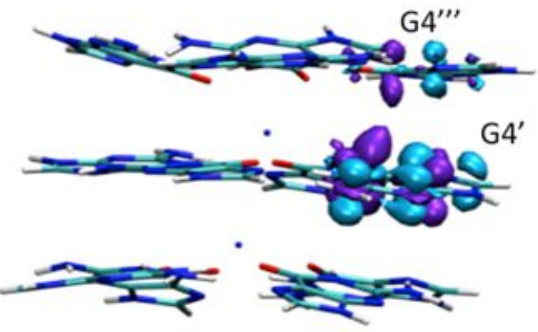

$\mathrm{S}_{4}$
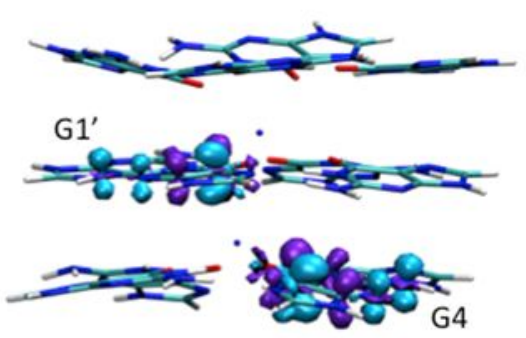

$S_{6}$
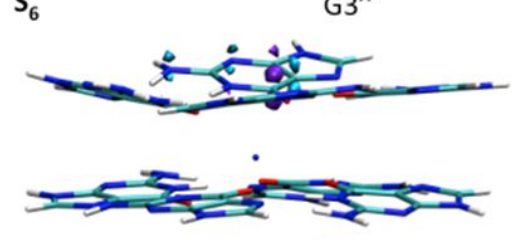

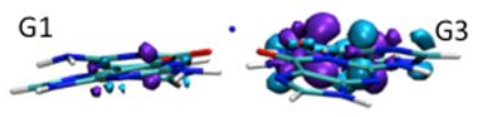

$\mathrm{S}_{8}$

G1" $\rightarrow$ का \&.8. $S_{10}$ G4" 
Figure SI-3. Electronic density difference $\left(\mathrm{S}_{n}-\mathrm{S}_{0}\right)$ corresponding to $\pi \pi *$ transitions associated with the La excited state at ground state minimum of Tel21/Na+ .
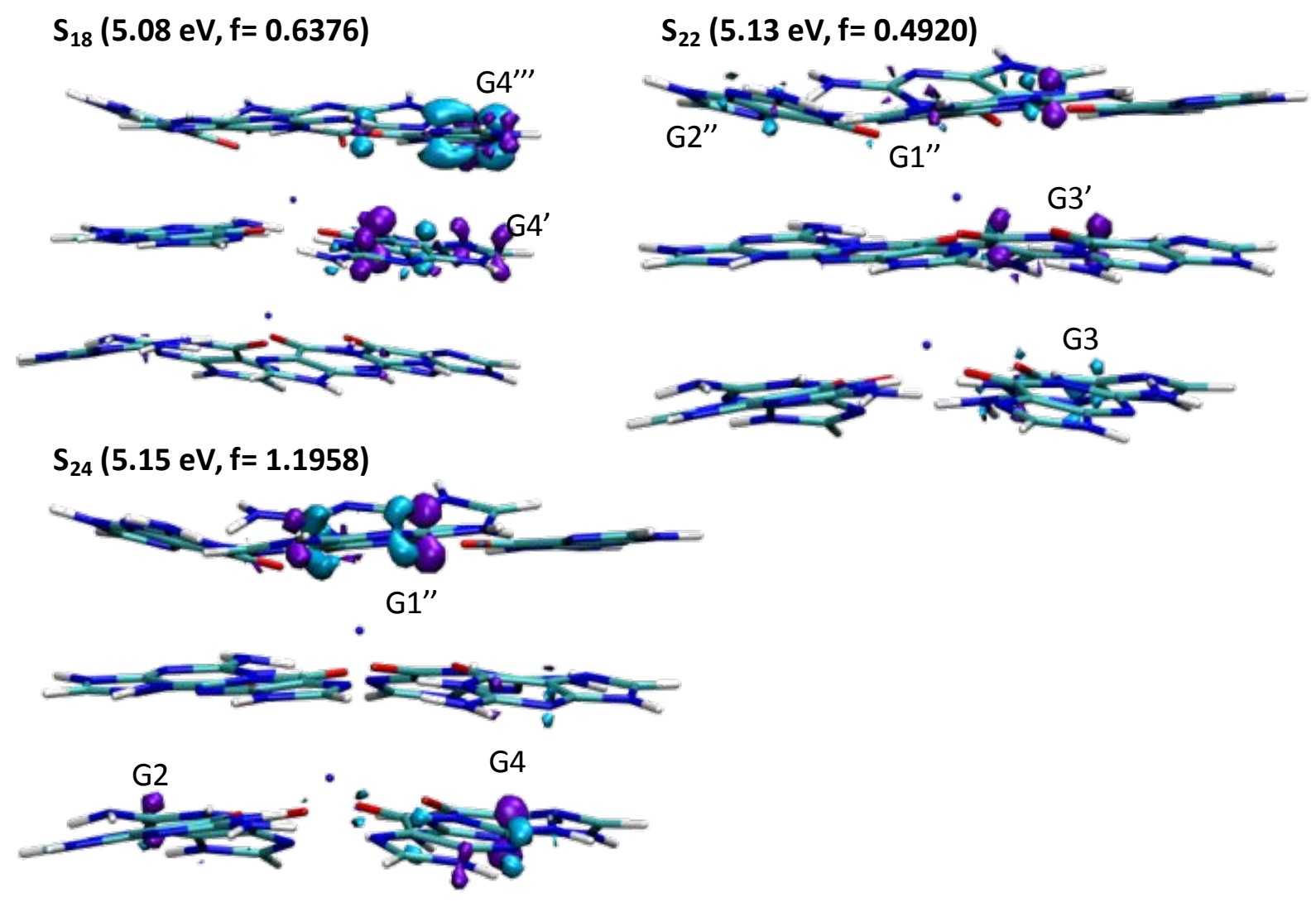

Figure SI-4. Electronic density difference $\left(S_{n}-S_{0}\right)$ corresponding to $\pi \pi *$ transitions associated with the Lb excited state at ground state minimum of Tel21/ $\mathrm{Na}^{+}$. 


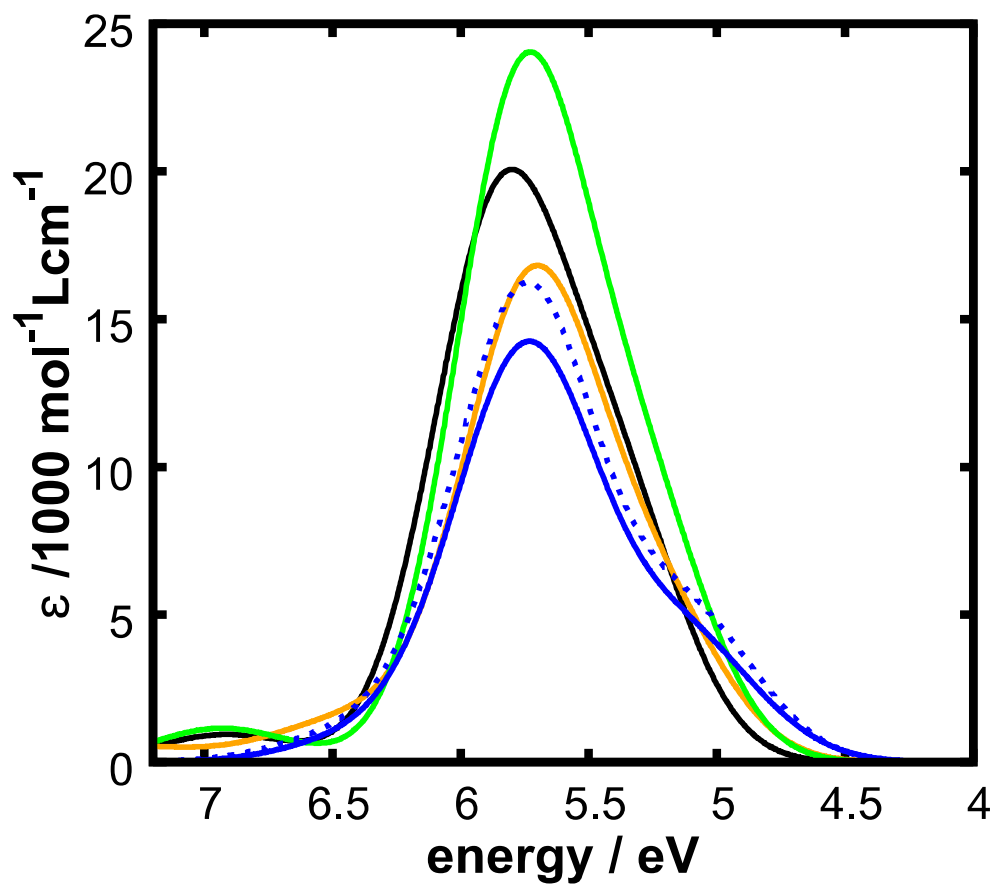

Figure SI-5. Absorption spectra compute for Model1 (blue solid line), Model2 (blue dashed line), GGG (orange), G tetrad (green) and sum of the monomers associated to Model2, i.e., 12 dGMP (black). The epsilon has been divided by the number of bases. 


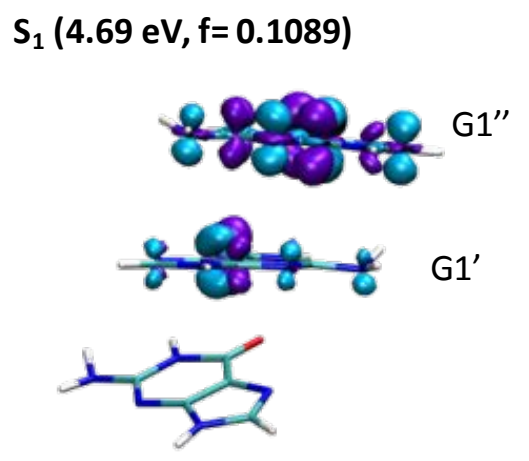

$S_{2}(4.71 e V, f=0.1193)$

$S_{7}(5.09 e V, f=0.1185)$

$S_{8}(5.29 e V, f=0.5728)$
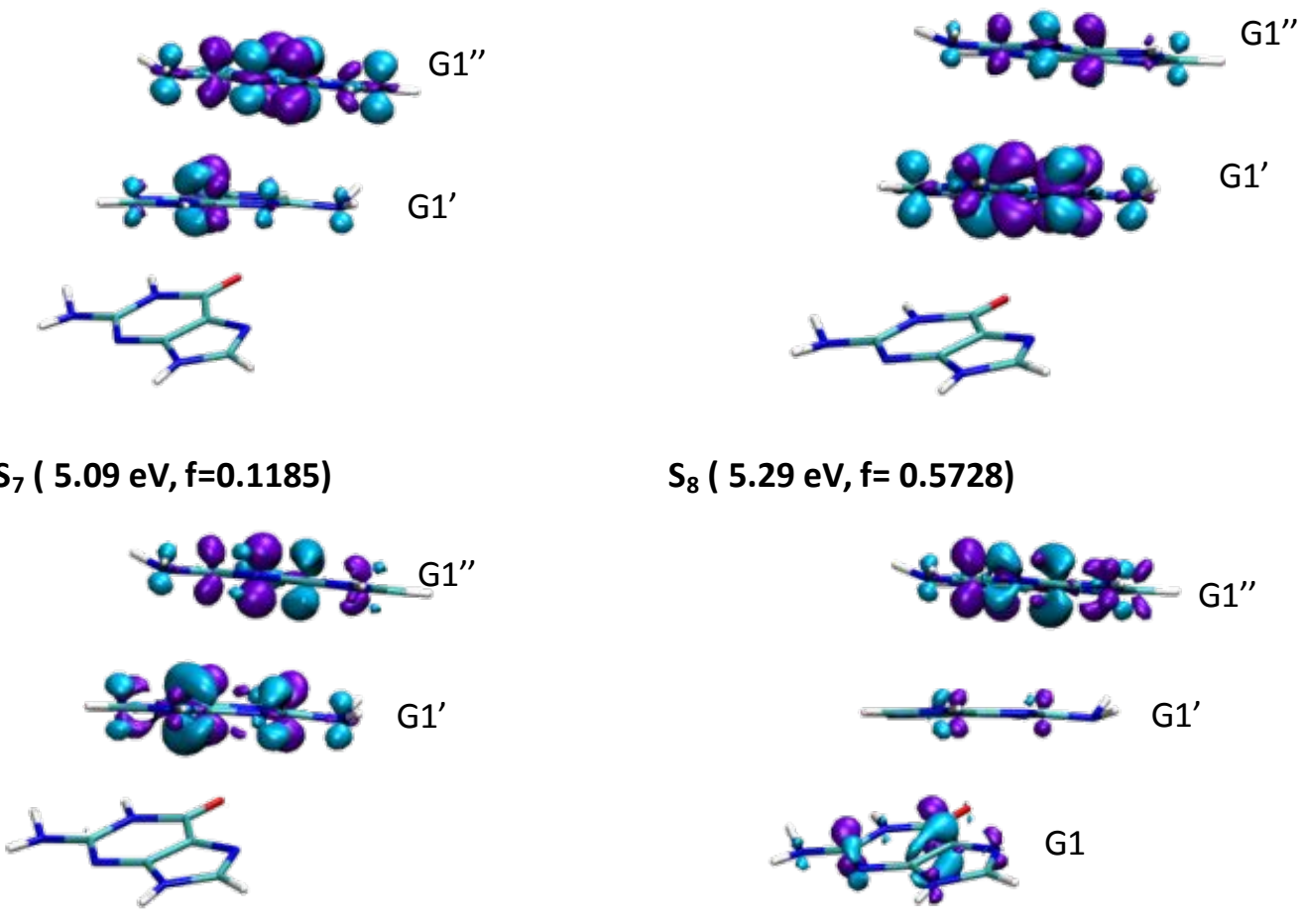

Figure SI-6. Electronic density difference $\left(S_{n}-S_{0}\right)$ corresponding to $\pi \pi *$ transitions associated with the La and Lb excited state at ground state minimum of the GGG strand

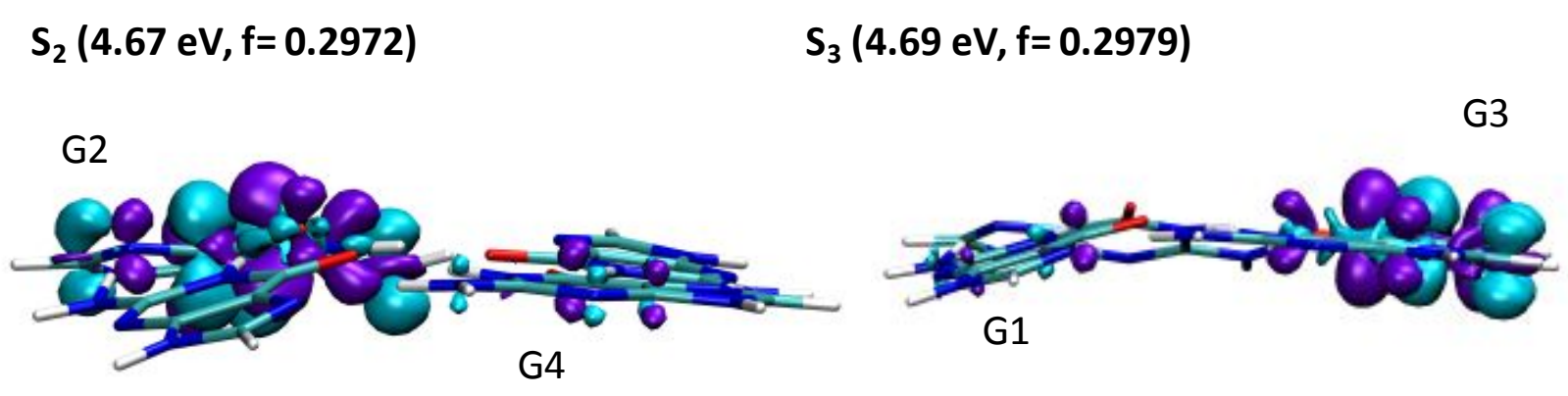

$S_{7}(5.16 e V, f=0.8850)$

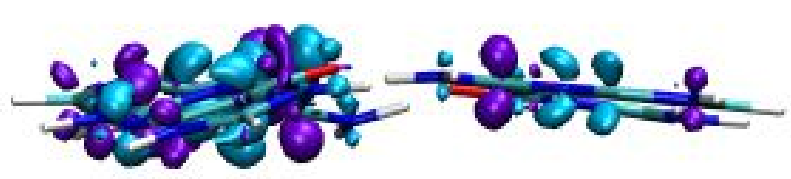

$S_{8}(5.17 \mathrm{eV}, \mathrm{f}=\mathbf{0 . 7 9 8 7})$

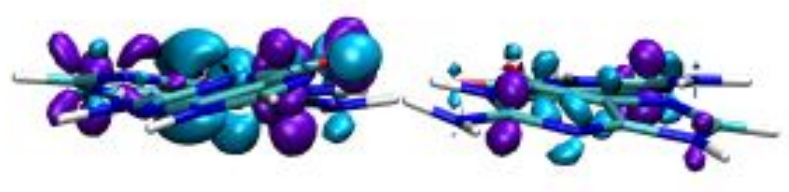


Figure SI-7. Electronic density difference $\left(S_{n}-S_{0}\right)$ corresponding to $\pi \pi *$ transitions associated with the La and Lb excited state at ground state minimum of the $G$ tetrad

Table SI-2. Adiabatic, vertical emission energies and oscillator strength of the different excited

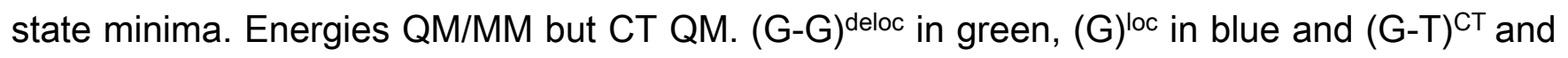
$(\mathrm{G}-\mathrm{G})^{\mathrm{CT}}$ in red.

\begin{tabular}{|c|c|c|c|c|c|}
\hline & $\Delta \mathrm{E} \mathrm{eV}$ & $\Delta \mathrm{E} \mathrm{eV}$ & $f$ & $\begin{array}{l}\text { Optimized } \\
\text { state }\end{array}$ & CT character \\
\hline $\begin{array}{l}\text { G3' ' } \rightarrow \text { G3'” * Ioc } \\
\text { G2' } \rightarrow \text { G2' * Ioc }\end{array}$ & $\begin{array}{l}4.58 \\
4.53\end{array}$ & $\begin{array}{l}4.44 \\
4.52\end{array}$ & $\begin{array}{l}0.16 \\
0.10\end{array}$ & $\begin{array}{l}S_{4} \\
S_{3} S_{5} S_{8}\end{array}$ & $\begin{array}{l}0.07 \\
0.06\end{array}$ \\
\hline G3" ' $\rightarrow$ G3' * deloc & 3.96 & 3.31 & 0.04 & $\mathrm{~S}_{1} \mathrm{~S}_{7}$ & 0.06 \\
\hline G4" ' $\rightarrow$ G4" * deloc & 4.28 & 3.31 & 0.03 & $S_{2} S_{6}$ & 0.07 \\
\hline G4"' $\rightarrow$ G4' CT & 5.11 & 4.26 & 0.01 & $\mathrm{~S}_{1}^{\mathrm{PBE}}$ & 0.68 \\
\hline G1'” $\rightarrow$ T CT & 4.06 & 3.52 & 0.00 & $\mathrm{~S}_{14}$ & 0.80 \\
\hline G2" ' $\rightarrow$ G2' * deloc & 3.94 & 3.35 & 0.03 & $S_{1}$ & - \\
\hline
\end{tabular}

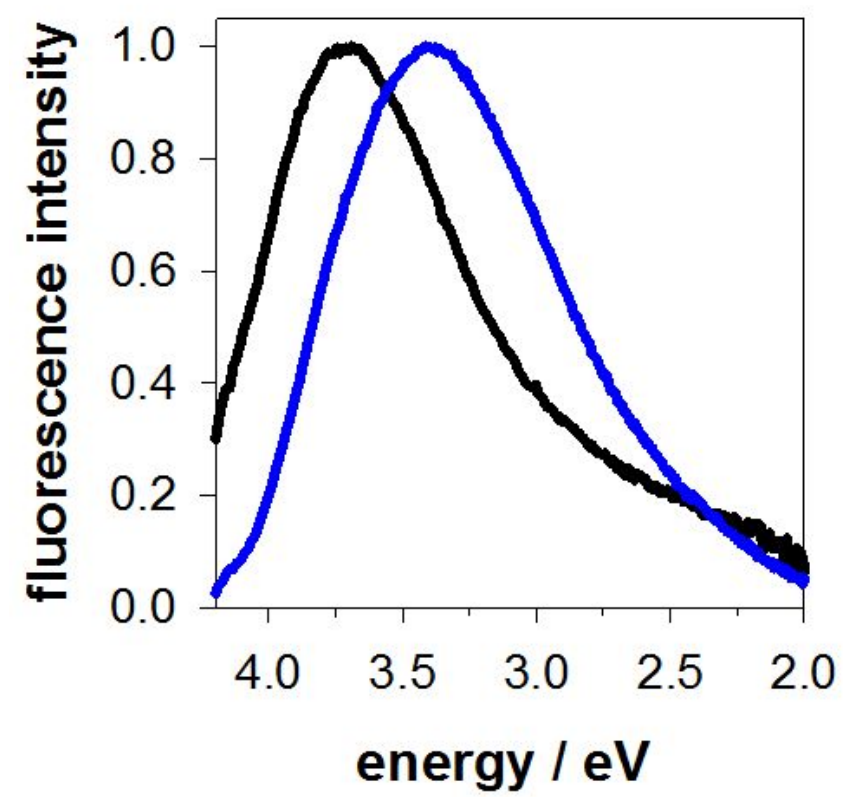

Figure SI-8. Normalized experimental fluorescence spectra of $\mathrm{TEL} 21 / \mathrm{Na}^{+}$(blue) and a mixture of mononucleotides dAMP, TMP and dGMP at ratio 4:2:1. 

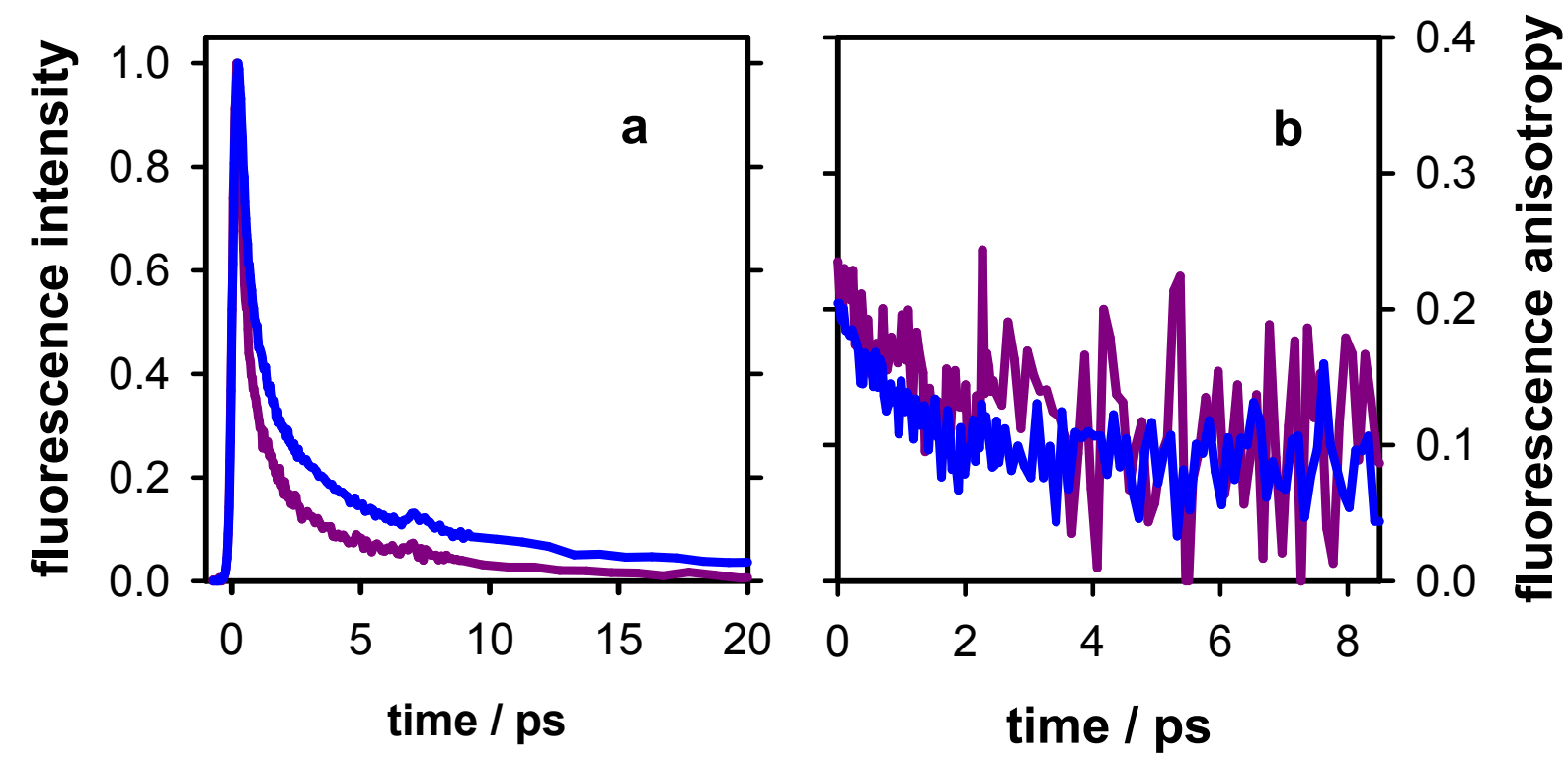

Figure SI-9. Normalized fluorescence decays (a) and fluorescence anisotropies (b) obtained for TEL21/ $\mathrm{Na}^{+}$at $315 \mathrm{~nm}$ (violet) and $340 \mathrm{~nm}$ (blue) by FU.
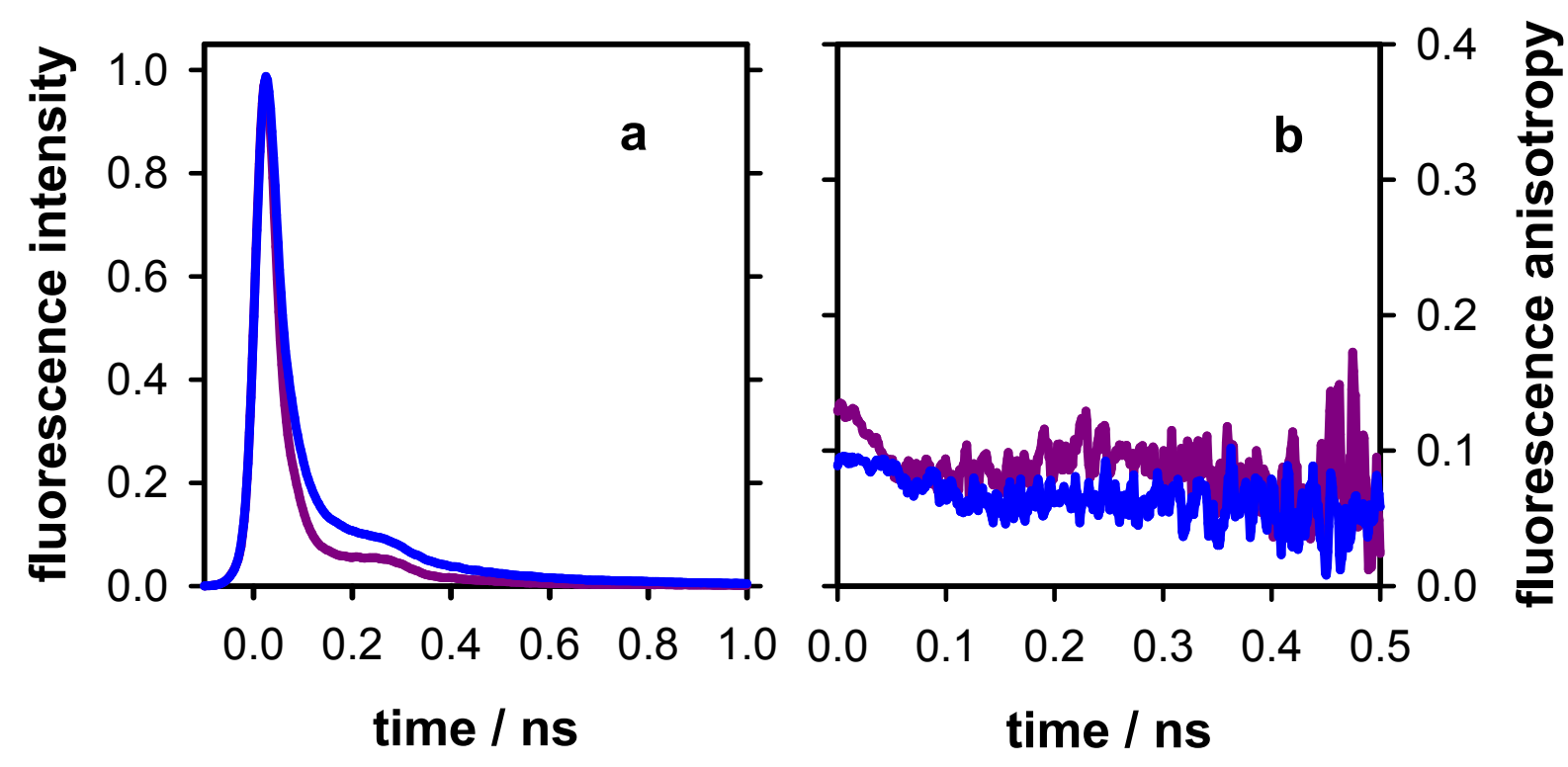

Figure SI-10. Normalized fluorescence decays (a) and fluorescence anisotropies (b) obtained for TEL21/Na+ at $315 \mathrm{~nm}$ (violet) and $340 \mathrm{~nm}$ (blue) by TCSPC. 


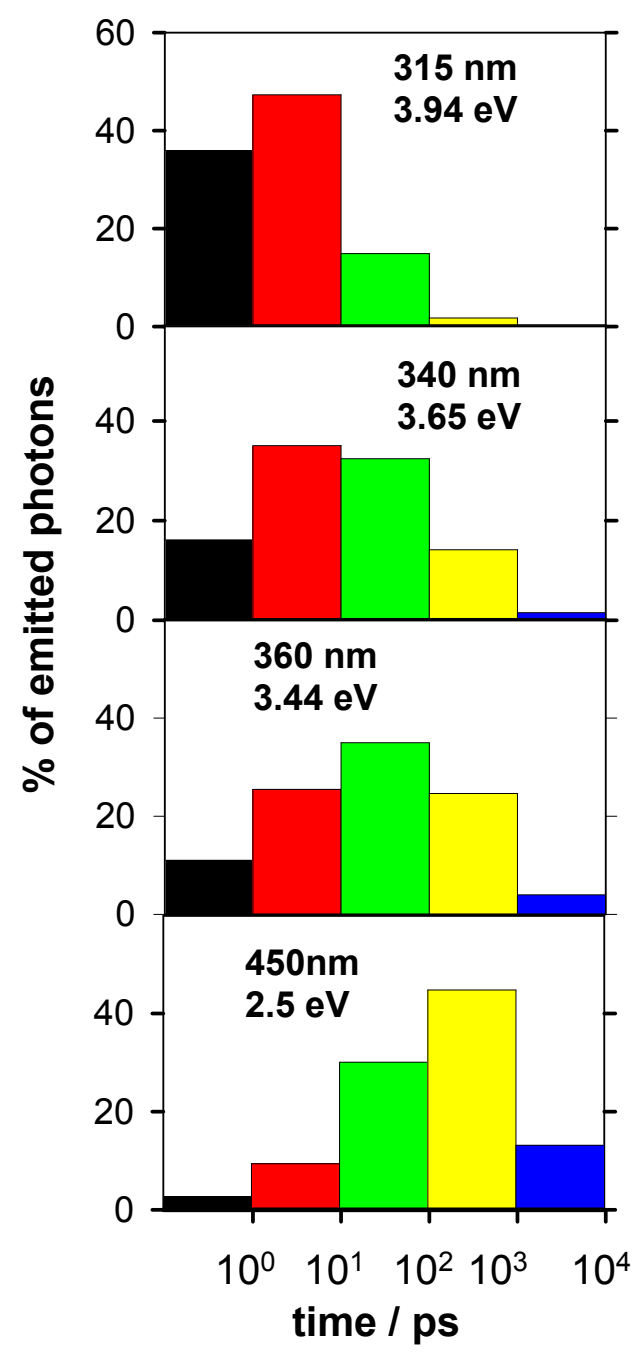

Figure SI-11. Percentage of emitted photons integrated per decade of time, derived from the joint fluorescence decays recorded by FU and TCSPC (Figure 2a). 


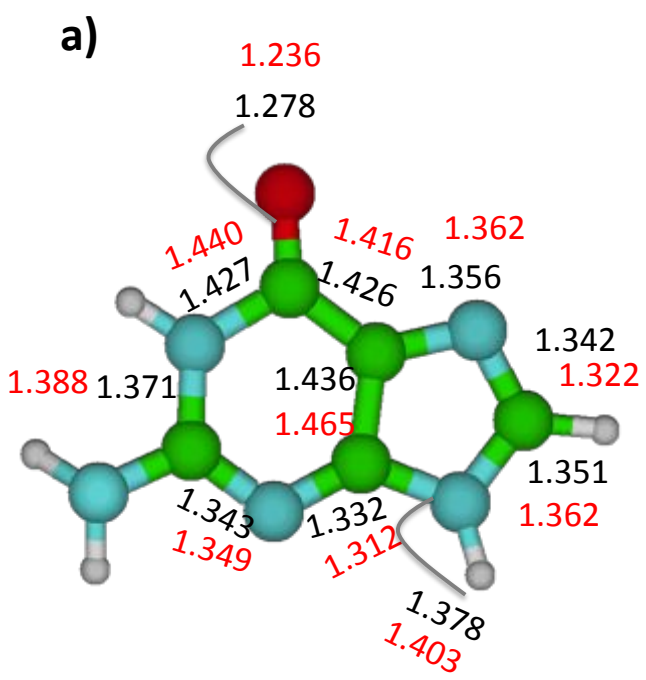

b)

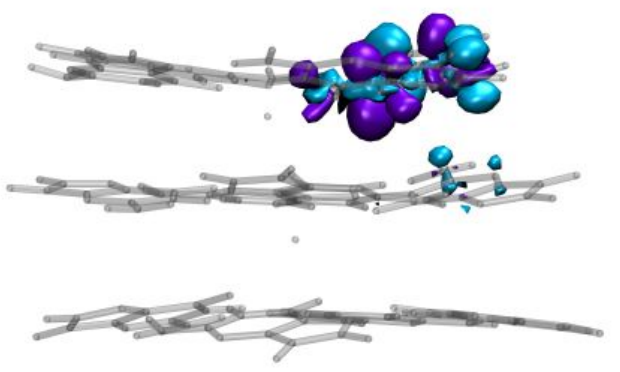

c)
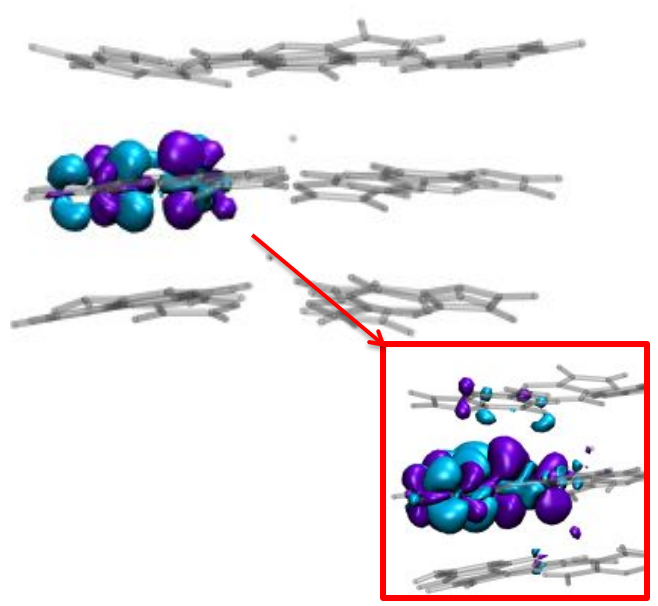

Figure SI-12. a) Bond distances at the optimized $\mathrm{S}_{1}$ minimum in dGMP (red) and Model2 (black). Density difference $\left(S_{n}-S_{0}\right)$ at the b) G3"G3"* and c) G2'G2'* minima; inset with smaller grid (0.0005).
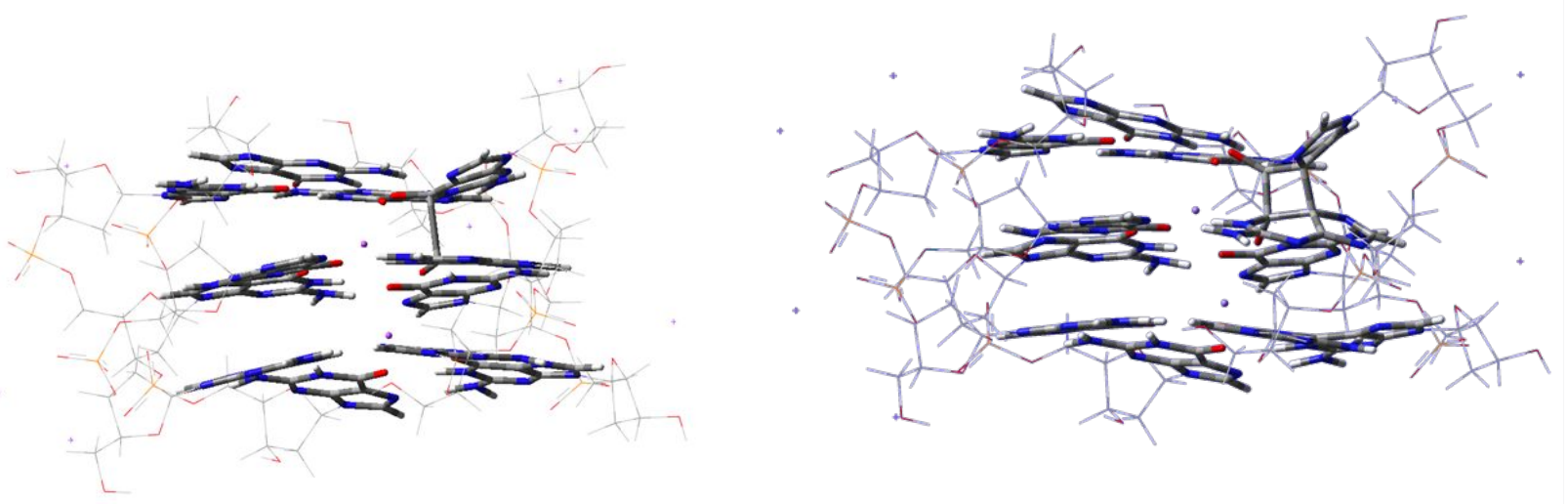

Figure SI-13. Geometry of the optimized (G-G) deloc (left) and (G-G)dimer (right) minima at the QM/MM level of theory. 


\section{References}

(1) Onidas, D.; Markovitsi, D.; Marguet, S.; Sharonov, A.; Gustavsson, T., Fluorescence properties of DNA nucleosides and nucleotides: a refined steady-state and femtosecond investigation. J. Phys. Chem. B 2002, 106, 11367-11374.

(2) Gustavsson, T.; Sharonov, A.; Onidas, D.; Markovitsi, D., Adenine, deoxyadenine and deoxyadenine 5 '-monophosphate studied by femtosecond fluorescence upconversion spectroscopy. Chem. Phys. Lett. 2002, 356, 49-54.

(3) Wang, Y.; Patel, D. J., Solution structure of the human telomeric repeat $\mathrm{d}\left[\mathrm{AG}_{3}\left(\mathrm{~T}_{2} \mathrm{AG}_{3}\right)_{3}\right] \mathrm{G}-$ tetratraplex. Structure 1993, 1, 263-282.

(4) Zhao, Y.; Schultz, N. E.; Truhlar, D. G., Design of density functionals by combining the method of constraint satisfaction with parametrization for thermochemistry, thermochemical kinetics, and noncovalent interactions. J. Chem. Theory Comput. 2006, 2, 364-382.

(5) Dargiewicz, M.; Biczysko, M.; Improta, R.; Barone, V., Solvent effects on electron-driven proton-transfer processes: adenine-thymine base pairs. Phys. Chem. Chem. Phys. 2012, 14, 8981-8989.

(6) Santoro, F.; Barone, V.; Improta, R., Absorption Spectrum of A-T DNA Unraveled by Quantum Mechanical Calculations in Solution on the (dA)2·(dT)2 Tetramer. ChemPhysChem 2008, 9, 2531-2537. (7) Improta, R., The excited states of $\pi$-stacked 9-methyladenine oligomers: a TD-DFT study in aqueous solution. Phys. Chem. Chem. Phys. 2008, 10, 2656-2664.

(8) Banyasz, A.; Martinez-Fernandez, L.; Balty, C.; Perron, M.; Douki, T.; Improta, R.; Markovitsi, D., Absorption of Low-Energy UV Radiation by Human Telomere G-Quadruplexes Generates LongLived Guanine Radical Cations. J. Am. Chem. Soc. 2017, 139, 10561-10568.

(9) Martínez-Fernández, L.; Pepino, A. J.; Segarra-Martí, J.; Jovaišaitè, J.; Vaya, I.; Nenov, A.; Markovitsi, D.; Gustavsson, T.; Banyasz, A.; Garavelli, M.; Improta, R., Photophysics of Deoxycytidine and 5-Methyldeoxycytidine in Solution: A Comprehensive Picture by Quantum Mechanical Calculations and Femtosecond Fluorescence Spectroscopy. J. Am. Chem. Soc. 2017, 139, 7780-7791.

(10) Conti, I.; Martínez-Fernández, L.; Esposito, L.; Hofinger, S.; Nenov, A.; Garavelli, M.; Improta, R., Multiple Electronic and Structural Factors Control Cyclobutane Pyrimidine Dimer and 6-4 Thymine-Thymine Photodimerization in a DNA Duplex. Chem. Eur. J. 2017, 23, 15177-15188. (11) Cornell, W. D.; Cieplak, P.; Bayly, C. I.; Gould, I. R.; Merz, K. M.; Ferguson, D. M.; Spellmeyer, D. C.; Fox, T.; Caldwell, J. W.; Kollman, P. A., A 2nd generation force-field for the simulation of proteins, nucleic acids, and organic molecules. J. Am. Chem. Soc. 1995, 117, 5179-5197.

(12) Dapprich, S.; Komaromi, I.; Byun, K. S.; Morokuma, K.; Frisch, M. J., A new ONIOM implementation in Gaussian98. Part I. The calculation of energies, gradients, vibrational frequencies and electric field derivatives. J. Mol. Struct.-Theochem 1999, 461, 1-21.

(13) Frisch et al., M. J., Gaussian 09, revision A.1; Gaussian, Inc.,Wallingford, CT, 2009.

(14) Tomasi, J.; Mennucci, B.; Cammi, R., Quantum mechanical continuum solvation models. Chem. Rev. 2005, 105, 2999-3093.

(15) Miertus, S.; Scrocco, E.; Tomasi, J., Electrostatic interaction of a solute with a continuum - A direct utilization of abinitio molecular potentials for the prevision of solvent effects Chem. Phys. 1981, 55, 117-129.

(16) Improta, R.; Scalmani, G.; Frisch, M. J.; Barone, V., Toward effective and reliable fluorescence energies in solution by a new state specific polarizable continuum model time dependent density functional theory approach. J. Chem. Phys. 2007, 127, 074504. 
(17) Improta, R.; Barone, V.; Scalmani, G.; Frisch, M. J., A state-specific polarizable continuum model time dependent density functional theory method for excited state calculations in solution. $J$. Chem. Phys. 2006, 125, 054103.

(18) Avila Ferrer, F. J.; Cerezo, J.; Stendardo, E.; Improta, R.; Santoro, F., Insights for an Accurate Comparison of Computational Data to Experimental Absorption and Emission Spectra: Beyond the Vertical Transition Approximation. J. Chem. Theor. Comput. 2013, 9, 2072-2082.

(19) Improta, R., Quantum Mechanical Calculations Unveil the Structure and Properties of the Absorbing and Emitting Excited Electronic States of Guanine Quadruplex. Chem. Eur. J. 2014, 20, 8106-8115.

(20) Improta, R.; Barone, V., Interplay between "Neutral" and "Charge-Transfer" Excimers Rules the Excited State Decay in Adenine-Rich Polynucleotides. Angew. Chem. Int. Ed. 2011, 50, 12016-12019. 